\title{
Lumped pseudo fission products during burnup step in MCNP5-ORIGEN coupling system
}

\author{
Hocine Benkharfia ${ }^{\mathrm{a}}$, Tahar Zidi ${ }^{\mathrm{b}}$, Mohamed Belgaid ${ }^{\mathrm{c}}$ \\ ${ }^{a}$ Nuclear Research Centre of Birine BP 180 Ain Oussera 17200, Djelfa, Algeria \\ ${ }^{b}$ Commissariat of Atomic Energy, 02 bd Frantz Fanon Street, BP 399, 1600 Algiers, Algeria \\ ${ }^{c}$ University of Science and Technology Houari Boumediene, 16111 Bab Ezzouar, Algiers, Algeria
}

Corresponding author: Hocine Benkharfia, Tel: +213 555207513, Fax: +21327874280

E-mail: bkfhocine@hotmail.com

\begin{abstract}
Depletion calculation and accurate inventory of fission products in a nuclear system are required for criticality, safety and spent fuel management. Actual trend is to use Monte Carlo methods. It is well know that the fission process produces a large number of nuclides, some of which have a significant impact on the nuclear properties of the core and its behavior. In this study, we propose to determine the influence of fission products on the behavior of the IAEA $10 \mathrm{MW}$ benchmark reactor. Even if nowadays we have powerful computing capability and we can solve the full system of fission products, such calculations are cumbersome and not needed because most of fission products have low absorption rates and therefore their precise concentrations calculation are not required. The practice is to identify and use only the nuclides which can have a significant absorption cross section.

From the entire fission products of the available fissionable actinides, 214 nuclides have been considered. Their selection was essentially based on their absorption rates. To carry out the calculation, 81 were treated explicitly and 133 were lumped into pseudo fission products.

A computational method has been developed for burnup and criticality calculations using MCNP5ORIGEN coupling scheme. The MIXE_ACE program was developed and incorporated within this coupling scheme in order to mix and rewrite in $A C E$ format the selected cross sections of the pseudo fission products for each burnup step. The mass weight of the constituent nuclides was used. The initial one group cross sections library for ORIGEN was generated using average flux spectrum in the core.

Using the above methodology, an estimation of $k_{\text {eff }}$ and cross sections during depletion calculations has been carried out for the IAEA $10 \mathrm{MW}$ reactor based on $\mathrm{UZrH1.6}$ fuel. The results are compared to those of ANL (Argonne National Laboratory), MCNP6 and other calculations by using selected fission products from WIMS library. Generally, the results are satisfactory but some discrepancies exist. The differences can be explained mainly by the nature of the fission products considered in the calculation and especially their cross sections.
\end{abstract}

Keywords: $M C N P 5$, ORIGEN, MCNP6, Fission products, $U Z r H 1.6$ fuel, End of cycle 


\section{Introduction}

Nuclear systems require a reliable inventory of fission products for safety and waste management purposes. The fission process in the nuclear fuel causes the accumulation of fission products. Some of these products have a significant impact on the nuclear properties of the core and its criticality. The main objectives of the depletion calculations are to provide as much details as possible of the composition of the irradiated fuel and to quantify the radioactive inventory for potential source terms determination (D. Lopez Aldama et al).

Nowadays, several burnup calculation codes have been developed, such as the Multi-Step Monte Carlo Burnup Code System MONTBURN (RSICC PSR-455) and MCNP-ORIGEN burnup calculation system code MCOR (Meiyin Zheng et al). Along the same lines an MCNP5-ORIGEN coupling system was developed to study the absorption effect of the fission products in light water reactors.

In this paper we perform criticality calculation at different burnup steps by considering the fission products that have an important impact. One lumped pseudo fission product is generally used for burnup calculations. In our approach, the cross sections of the pseudo fission products are calculated in each burnup step to take into account the variation of the concentration of each nuclide during burnup step by the means of $M I X E_{-} A C E$ program, which can be used as a new module in $M C N P 5-O R I G E N$ coupled system. In this way, the inventory of fission products is established in each fuel assembly to get the end of cycle $(E O C)$ with more precision. The developed scheme was applied to the initial fuel load (BOC, Beginning of Cycle) of IAEA $10 \mathrm{MW}$ reactor which uses an $U Z r H 1.6$ fuel $\left(\mathrm{N}_{\mathrm{H}} / \mathrm{N}_{\mathrm{Zr}}=1.6\right)$.

Since the MCNP6 code (Denis B. Polowitz) allows burnup calculations, and in order to verify the obtained results and to validate the developed coupling scheme, burnup calculations have been carried out by MCNP6 code using "burn" option with "tier3" fission products contents which have been treated explicitly.

\section{Computational methods}

\subsection{Cross section processing}

More than 450 fission products and the important fissionable nuclides were processed by using the comprehensive NJOY code system (RSICC PSR-480). NJOY is a modular computer code system used for processing and converting evaluated nuclear data in $E N D F$ format into different types of data libraries that can be used in several applications, the $99+$ version of the code was used. Evaluated nuclear data files for most nuclides are extracted from ENDF/B-VII (ENDF/B-VII.1) and from TENDL2012 (Koning et all) for some nuclides. To produce point-wise data at $300{ }^{\circ} \mathrm{K}$ for the considered nuclides in PENDF files, the adapted sequence is the following: MODER (converts ENDF "tapes" back and forth between formatted and blocked binary modes), RECONR 
(reconstructs energy-dependent pointwise cross sections), BRODR (Doppler-broadens), HEATR (heat production cross section), GASPR (gas production cross section), THERMR (scattering in the thermal energy range) and PURR (unresolved-region probability tables for the MCNP code) modules.

To produce $A C E$ formatted files that are not available in MCNP5 library, the ACER module has been used. For isotopes in isomeric states, the Zaid suffix has been changed ( +50 to the ground state), (Data Bank 2006)

\subsection{Selection of the fission products}

There are more than 1300 fission product yield data for each fissionable nuclide in the $E N D F / B-V I I$ fission product yield file (Choong et all). In this study, from the fission products processed by NJOY code, only the nuclides that affect the criticality of the core during the fuel depletion have been taken into consideration, even if their radioactive decay constants are short. In this way, the selection of the nuclides was essentially based on their absorption rates (fission yields and abserption cross sections). A total of 214 nuclides were identified, 81 nuclides were represented explicitly and 133 nuclides have been lumped into the pseudo fission product More precisely, only the highly absorbing fission products which satisfy $\gamma \sigma>1.2 \times 10^{-8}$ barns, where $\gamma$ and $\sigma$ are the fission yield and the absorption cross section of the considered nuclides respectively (A.Trkov), have been selected. This assumption helps us to identify 214 fission products as important nuclides having significant effects on the criticality calculation.

In general, the fission products, which have large reactivity effects, are treated explicitly, and others are treated as a pseudo fission products.

Various fission product models were obtained by varying the above criterion and therefore the number of nuclides which must be treated explicitly. The models were carefully studied and tested. The condition is that the cross sections of the pseudo nuclide were calculated assuming infinite dilution but should provide the same criticality calculation if all fission products are treated explicitly. As a result of this study, our model was formed of 81 nuclides which were treated explicitly and 133 nuclides which were lumped into one pseudo nuclide as shown in (Table1).

Insert Table 1. 


\subsection{Lumped fission products cross sections}

To generate cross sections for pseudo fission products used by $M C N P 5$ code, $M I X E \_A C E$ program was developed in order to mix and rewrite in $A C E$ file cross sections for total, diffusion, (n,2n), $(\mathrm{n}, 3 \mathrm{n}),\left(\mathrm{n}, \mathrm{n}^{\prime}\right),(\mathrm{n}$, continuum), $(\mathrm{n}, \gamma),(\mathrm{n}, \mathrm{p})$ and $(\mathrm{n}, \alpha)$ reactions. Cross-sections of the pseudo fission products were calculated by adopting the mass weights of the constituent nuclides, linear-linear interpolation was applied. The mass weights were extracted from ORIGEN output for each burnup step. MIXE_ACE program uses, as input, the generated binary file, which contains the pertinent cross section for the all 214 selected fission product nuclides. This file was prepared from PENDF files

\section{MCNP5-ORIGEN coupling system}

A computational method has been developed for burnup calculation. The method uses the flux and reaction rates calculated by the General Monte Carlo N-Particle Transport Code MCNP5 (Jeremy and al, 2003), developed by Los Alamos National Laboratory (LANL), and the ORIGEN computer code (M.J. Bell) intended to determine the fuel isotopic composition after irradiation. The ORIGEN code, developed for depletion calculation, uses zero-dimensional geometry and one group cross section. The code did not take account of spatial or resonance self-shielding effects or changes in neutron spectra other than embodied initially which is in accordance with our proposed scheme. For each burnup step in our coupling system six tasks are performed Fig 1:

1) Criticality calculation of the considered reactor core for initial concentrations by using MCNP5 code. We obtain the multiplication factor, deposited power and one energy group average flux in each fuel assembly as well as the reaction rates for the considered nuclides of the fuel.

2) One group cross sections calculations using the average flux and reaction rates for each fuel assembly from the earlier $M C N P$ output.

3) ORIGEN library updating by using the new obtained cross section sets.

4) New isotope concentrations calculations for burnup time-step, including a predictor-corrector scheme; hence a twice run of ORIGEN is performed for each fuel assembly.

5) Cross sections $(X S)$ mixing for lumped fission product using the new concentrations.

6) $A C E$ file updating for the pseudo fission product.

This sequence is repeated in an automatic way until the end of cycle $\left(E O C, K_{e f f} \leq 1\right)$, or until the number of prefixed burnup cycles is reached.

Insert Fig. 1. 


\section{Benchmark problem modeling}

\subsection{Core description}

The IAEA-10 MW reactor is based on $U Z r H 1.6$ fuel core. It consists of $6 \times 6$ grid core, containing 30 fuel cluster elements which have a 16 fuel rods in $4 \times 4$ square array, 4 control rods fully withdrawn from the core and two irradiation channels as shown in Fig.2. In this study, we consider the case when the core is reflected by light water on the 4 sides. The cluster is contained within rectangular aluminum shroud with inner dimensions forming a $6.805 \mathrm{~cm}$ square and two different thicknesses of $0.384 \mathrm{~cm}$ and $0.579 \mathrm{~cm}$. The fuel rod is $1.377 \mathrm{~cm}$ in diameter and active length of $55.88 \mathrm{~cm}$, it is cladded with $0.041 \mathrm{~cm}$ thickness of Incoloy 800. Detailed specifications of this benchmark are described in Table 2 (TECDOC 233).

Insert Fig. 2.

Insert Table 2.

\subsection{ORIGEN library}

For the UZrH1.6 benchmark core, a specific MCNP5 model is adopted considering only fresh fuel for all fuel assemblies and F4 tally in 450 energy intervals. This model is used to calculate average neutron energy spectrum. MCNP5 provides the average flux between the two ends of each interval. To calculate the flux values in the considered points, we can suppose the flux as constant or linear between the two points. The results of the two assumptions are very close to each other and the curve of the average flux is presented in Fig. 3.

Insert Fig. 3.

It is seen that the flux is nearly Maxwellian between 0 and $0.55 \mathrm{eV}$ and is linear along the interval [0.55 eV, $100 \mathrm{keV}]$.

One group average cross sections were calculated for more than 450 fission products and important fissionable nuclides. The calculations were carried out by averaging the desired cross sections in $P E N D F$ files produced by NJOY over the considered flux spectrum. The light water ORIGEN library was subsequently updated by the calculated one group cross sections. 


\subsection{MCNP Model for burnup calculation}

A three dimensional model of the benchmark reactor was set up considering each fuel cluster separately Figs. 4 and 5. In total, 30 fuel regions were considered, each region and each nuclide have been treated explicitly, and pertinent tallies have been requested to calculate average flux, average deposited energy and reaction rates for (n,fission) and (n, $\gamma)$ reactions from 0 to $20 \mathrm{MeV}$ in concordance with the one group ORIGEN depletion calculations. Reactions of types (n,p), (n, $\alpha)$, $(\mathrm{n}, 2 \mathrm{n})$ and $(\mathrm{n}, 3 \mathrm{n})$ were not considered in $M C N P$ calculation because their variation with fuel depletion are very small compared to the fission and capture cross sections. These reactions were supposed to be constant in ORIGEN calculations during burnup steps. MCNP6 calculation is performed using the same model with burnup option and tier3 fission products contents.

Insert Fig. 4.

Insert Fig. 5.

\section{Results and discussions}

\subsection{MCNP modeling Validation}

In the $K C O D E$ calculations, a constant starting source was used. 300 inactive cycles were run, followed by 900 active cycles, each of 3,000 histories. The F7 tally was used to obtain average deposited energy in each fuel assembly and F4 tally for flux and reaction rates. The statistical error is less than $2 \%$ for flux, deposited energy, fission and capture rates.

To validate the $M C N P$ model a comparison of the effective multiplication factor $k_{\text {eff }}$ for initial core with $A N L$ and MCNP6 results was done. It should be noted that the keff for xenon equilibrium state in our calculation is estimated by considering the 214 fission products in the core after one hundred hours. The results are given in the following table 3 :

\section{Insert Table 3.}

The results appear to be satisfactory for the beginning of cycle $(B O C)$ and the reactor core model can be used to the study of the burnup for establishing the inventory of the fission products at the end of cycle. For Xenon equilibrium state, only the Xe-135 is considered in Argon National Laboratory results. 


\subsection{Burnup calculations:}

Burnup calculations were performed using the ORIGEN code. The code was modified to allow automated calculation of each fuel assembly. ORIGEN uses the flux and cross sections such as calculated by MCNP5, and provides, as result, the inventory of the actinides and a very large number of fission products. The concentrations of the 214 selected nuclides with some other necessary actinides such as U-235, U-236, U-238, Pu-239, Pu-240, Pu-241 and Pu242, have been extracted and employed to build the new input file for $M C N P 5$ code.

Considering the reactor core model, the MCNP5-ORIGEN coupling system was executed for UZrH1.6 benchmark to calculate the effective multiplication factor as a function of burnup. The results are presented on Figs. 6 and 7.

In a first calculation, all 214 fission products (FPs) were treated explicitly for our selection. The same work was carried out for the 135 fission products of the WIMS library (D. Lopez Aldama et all). These calculations were done to verify the results without considering the pseudo fission products. For MCNP6 calculation, any pseudo fission product was considered Fig.6

Insert Fig. 6.

In this case, we note that a good agreement between our results (214 FPs) and those of $A N L$, the differences become significant when the concentrations of fission products become very important. Probably, some fission products and especially those in isomeric state are not considered by $A N L$ calculation. These nuclides present substantial absorption cross sections and they are not considered in WIMS library and tier3 of MCNP6, which explains the large discrepancies in the results compared to our calculation.

Moreover, the obtained results with the nuclides of WIMS library are very close to those of MCNP6, even in "tier3" there are more fission products, but their cross sections are not very important and the variation of power with burnup is not considered in the calculation. Therefore, there is an important difference between WIMS fission products selection results and those of $A N L$. This difference can be explained by the absence of some fission products that affect the criticality calculation of the core in the WIMS library.

Repeating now the same calculation of keff with burnup using the pseudo fission products; in each burnup step, the 133 nuclides were lumped into a one pseudo fission product for our selection and 79 nuclides for WIMS library. The other nuclides were treated explicitly. The results are shown in Fig. 7

Insert Fig. 7. 
When we consider a pseudo fission product in the calculation, we note a good agreement of the results compared to those when all fission products are treated explicitly as shown in Fig. 7, particularly in the beginning of life. From 5000 MWD, the difference is small which can be neglected. This variation can be explained by the presence of some nuclides with absorption cross sections relatively important. On the other side, the results obtained when the pseudo fission product is introduced in the selected nuclides of WIMS library, coincides with those calculated without mixing.

The cross sections of the pseudo fission product were calculated assuming infinite dilution and adopting the mass weights of the nuclides without any self-shielding corrections in the pseudo composition. This assumption is used to identify the nuclides which compose the pseudo fission products. More than the highly absorbing nuclides are added to the pseudo fission product, more the results become unacceptable and the self-shielding correction will be necessary. Generally, the difference between the results of the criticality calculation is due essentially to the cross sections of the considered fission products, which should be carefully chosen.

In the following figure, we present $(\mathrm{n}, \gamma)$ one group absorption cross section as a function of burnup of the pseudo fission products calculated by MCNP5 for 133 and 79 nuclides.

\section{Insert Fig. 8.}

To show the importance of the mixing cross sections of the lumped fission products in each burnup step, we present $(n, \gamma)$ cross section of the pseudo fission products in the beginning and the end of load of the core for 133 nuclides and 79 fission products of WIMS library calculated by MIXE_ACE program in the following figures:

\section{Insert Fig. 9.}

Insert Fig. 10.

For WIMS library Fig. 10, the difference between cross sections of the pseudo fission products is very small and can be neglected. In this case, we can consider only the cumulative yields data of the fission products for weighting cross-sections. But for our selection Fig. 9, the difference is important, in particular in the thermal and epithermal regions, which can be easily explained by the significant change in the concentrations of different fission products during fuel depletion, and especially the nuclides representing important absorption cross section Fig. 8. 


\section{Conclusion}

The fission products are important for analyzing the core characteristics behavior with burnup and the determination of the end of cycle. The identification of the nuclides that affect the criticality of the core is very important, even if these nuclides have a short radioactive decay constant. It is customary to use pseudo fission products, their cross sections are calculated as weighted averages of the constituent's nuclides cross sections. The results obtained by the selection of 214 fission products are satisfactory and more realistic compared to other work (ANL). Also it is to be noted that the results with WIMS library nuclides are very close to those of MCNP6, which can be explained by assuming that the average absorption cross section of the fission products is in the same order of magnitude. In our opinion, the use of the MCNP5-ORIGEN coupled scheme and $M I X E \_A C E$ program for mixing cross section of the pseudo fission product in each burnup step gives more accurate results.

\section{Acknowledgement}

We thank the DOE for allowing the use of MCNP versions through the GTRI cluster.

\section{References}

A.Trkov, Program AVRFPY, Institute J.Stefan, Ljubljana, Slovenia (1995), Upgrade to support INTER 6.12 output (2011).

A.Trkov, D.L.Aldama, "Definition of the pseudo fission product data for reactor calculations“, Nuclear Energy in Central Europe (international conference 1999), Jozef Stefan Institute, Ljubljana (1999) 57-62.

A.J. Koning et All, TALYS-based Evaluated Nuclear Data Library, Incident-Neutron Data 2012

Choong-Sup Gil, Do Heon Kim, and Jonghwa Chang, Group Constants Generation of the Pseudo Fission Productsfor Fast Reactor Burnup Calculations, CP769, International Conference on Nuclear Data for Science and Technology, American Institute of Physics; 2005.

Denise B. Polowitz et all, MCNP6 Users Manual - Code Version 6.1.1beta, LA-CP-13-00634, Rev. 0 (May 2013).

D. Lopez Aldama, F. Leszczynski, A. Trkov, WIMS-D Library Update, Final report of a coordinated research project, IAEA 2013.

Data Bank, OECD NEA Processing of the JEFF-3.1 Cross Section Library into a Continuous Energy Monte Carlo Radiation Transport and Criticality Data Library, NEA/NSC/DOC(2006)18.

ENDF/B-VII.1 U.S. Evaluated Nuclear Data Library, Incident-Neutron Data, issued in 2011 
H. Jeong and S.H. Chang, Estimate of the fission products, actinides and tritium of HTR-10, Nuclear Engineering and Technology, vol 41 No 5, June 2009

IAEA "IAEA Research Reactor Core Conversion from the use of high-enriched uranium to the use of low enriched uranium fuels Guidebook”. IAEA-TECDOC-233, 1980.

Jeremy E., "MCNP5 - A General Monte Carlo N-Particle Transport Code, Version 5", LA-UR-03-1987, LANL, Los Alamos, NM, 2003.

Meiyin Zheng et all, Development of MCNP-ORIGEN burnup calculation code system and its accuracy assessment, MCOR, volume 63, January 2014, pages 491-198.

M.J. Bell, 'ORIGEN-The ORNL Isotope Generation and Depletion Code', ORNL-4628, RSIC.

RSICC PSR-455, MONTEBURNS 2.0, An Automated, Multi-Step Monte Carlo Burnup Code System, July 2003.

RSICC PSR-480, NJOY99.0 Code System for Producing, Pointwise and Multigroup Neutron and Photon Cross Sections from ENDFB Data, March 2000.

\section{Table Captions}

Table 1: Fission product nuclides, in bold those treated explicitly

Table 2: Benchmark specifications

Table 3: Eigenvalues for initial core

\section{Figure Captions}

Fig.1. Burnup calculation in MCNP5-ORIGEN computational method

Fig.2. XY Model for initial core, core burnup studies on $10 \mathrm{MW}$ reactor using UZrH1.6 LEU Fuel

Fig.3. Average neutron flux spectrum in the fuel region

Fig.4. Radial Tally Cells

Fig.5. Axial Tally Cells

Fig.6. Variation of Keff with burnup (All FPs treated explicitly)

Fig.7. Variation of Keff with burnup considering the pseudo fission products

Fig.8. $(n, \gamma)$ one group cross section of the pseudo fission products

Fig.9. $(n, \gamma)$ cross section in the beginning and the end of cycle of the pseudo fission product (133 nuclides)

Fig. 10. $(\mathrm{n}, \gamma)$ cross section in the beginning and the end of cycle of the pseudo fission product (79 nuclides) 
Fig. 1.

Preparation of MCNP5 input files:

Reactor model with initial concentrations

ACE file for pseudo fission products (PFP)

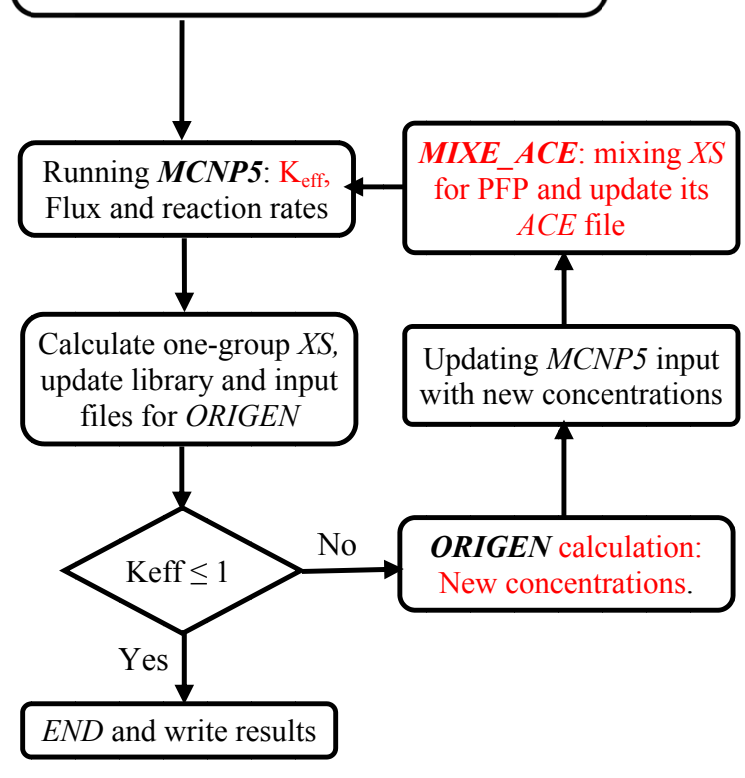

Fig. 2.

\begin{tabular}{|c|c|c|c|c|c|c|c|}
\hline Water & Fuel & Fuel & $C R-1$ & Fuel & Fuel & Fuel & Water \\
\hline Water & Fuel & Fuel & Fuel & Fuel & Fuel & Fuel & Water \\
\hline Water & Fuel & Fuel & Fuel & Water & Fuel & CR-3 & Water \\
\hline Witer & Fuel & $C R-2$ & Fuel & Fuel & Fuel & Fuel & Water \\
\hline Water & Fuel & Fuel & Fuel & Fuel & CR-4 & Fuel & Water \\
\hline Water & Fuel & Fuel & Fuel & Fuel & Fuel & Water & Water \\
\hline
\end{tabular}


Fig. 3.

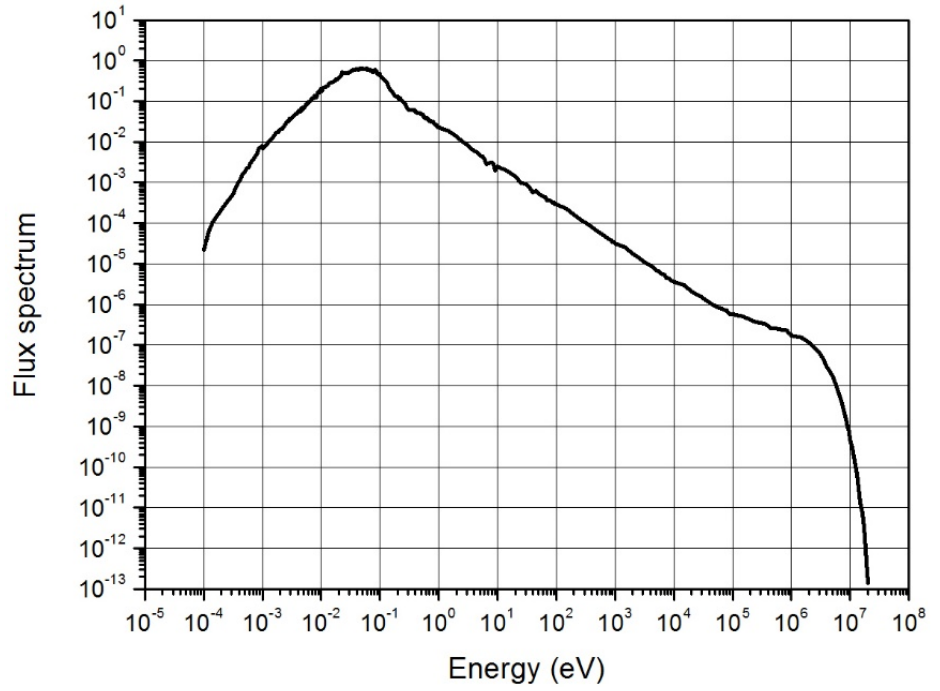

Fig. 4.

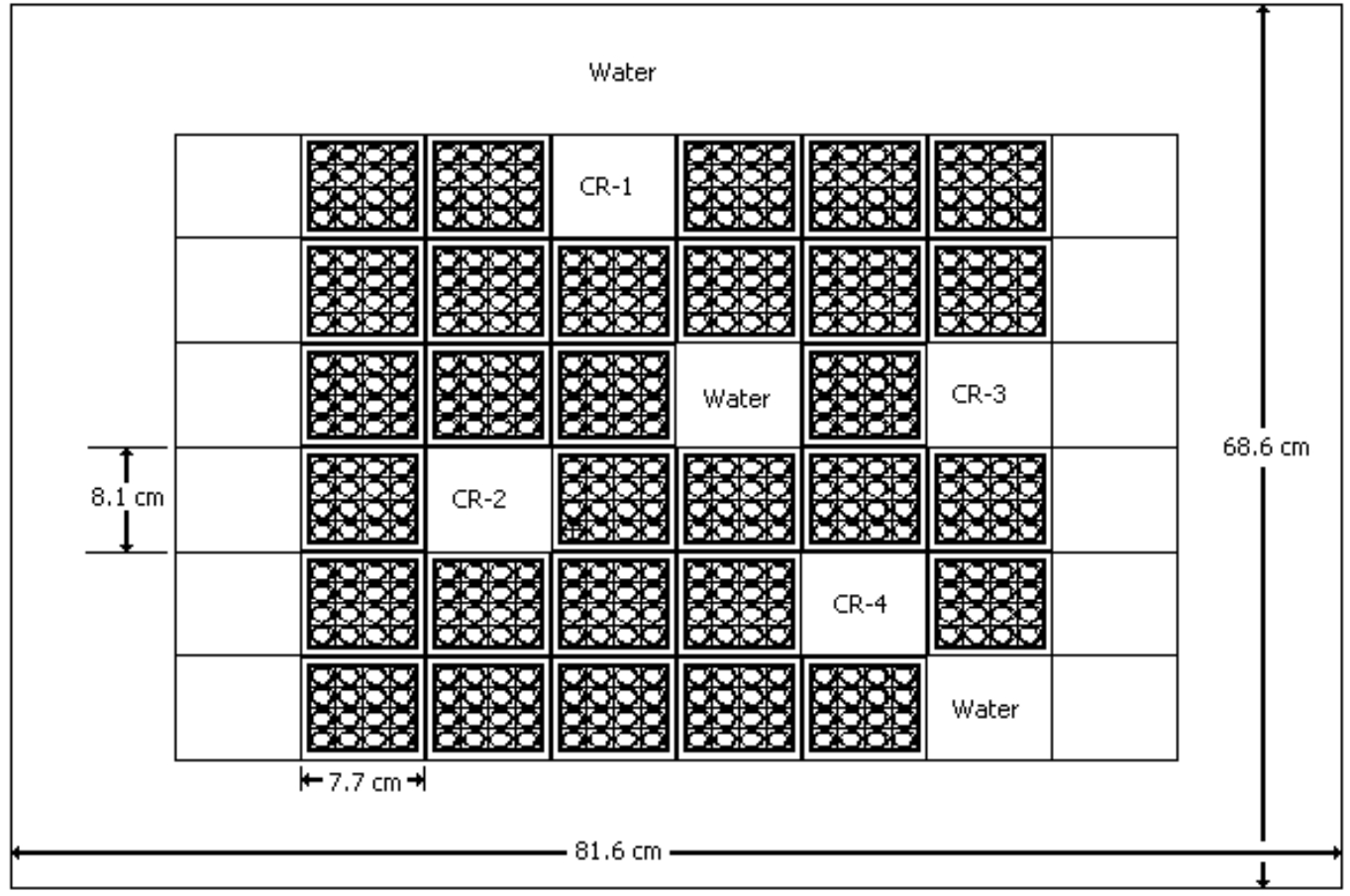


Fig. 5.

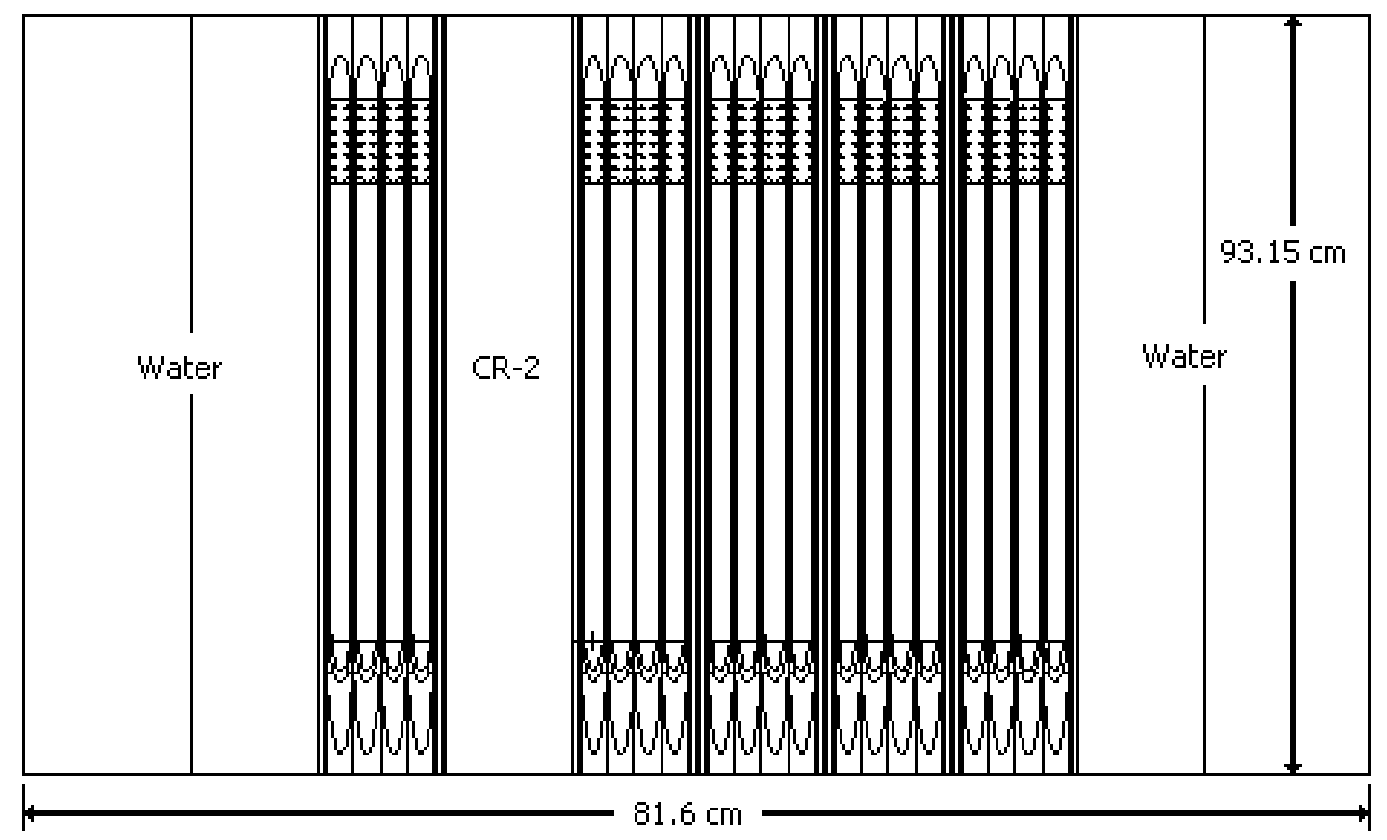

Fig. 6.

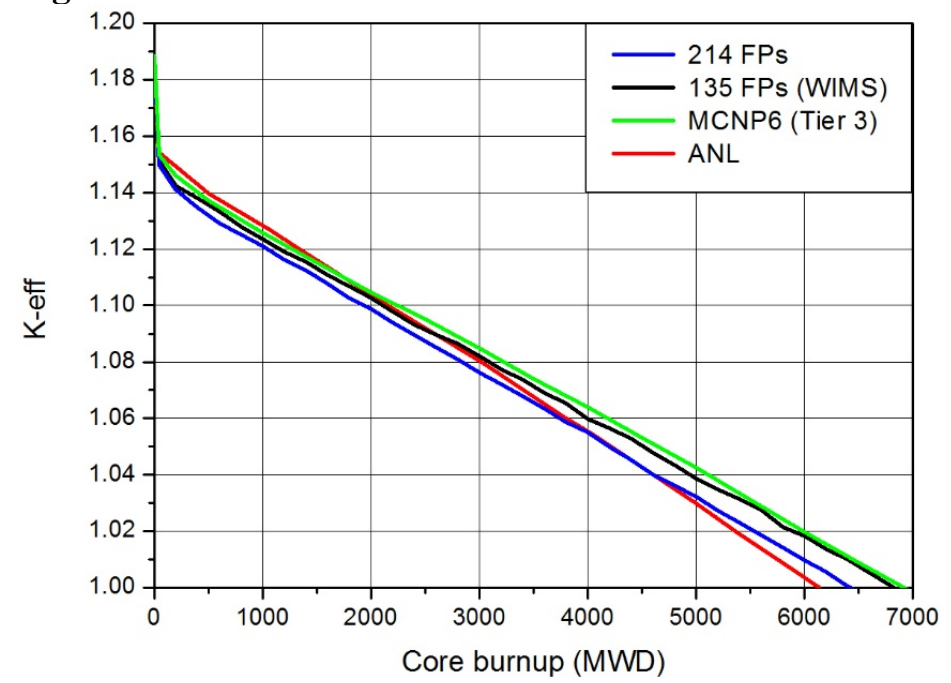


Fig. 7.

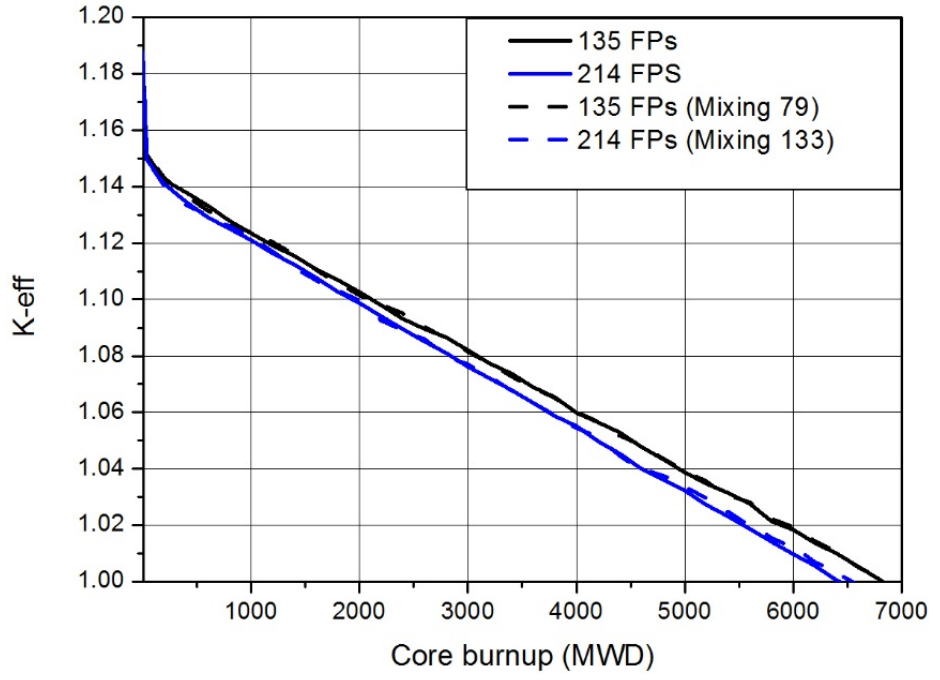

Fig. 8.

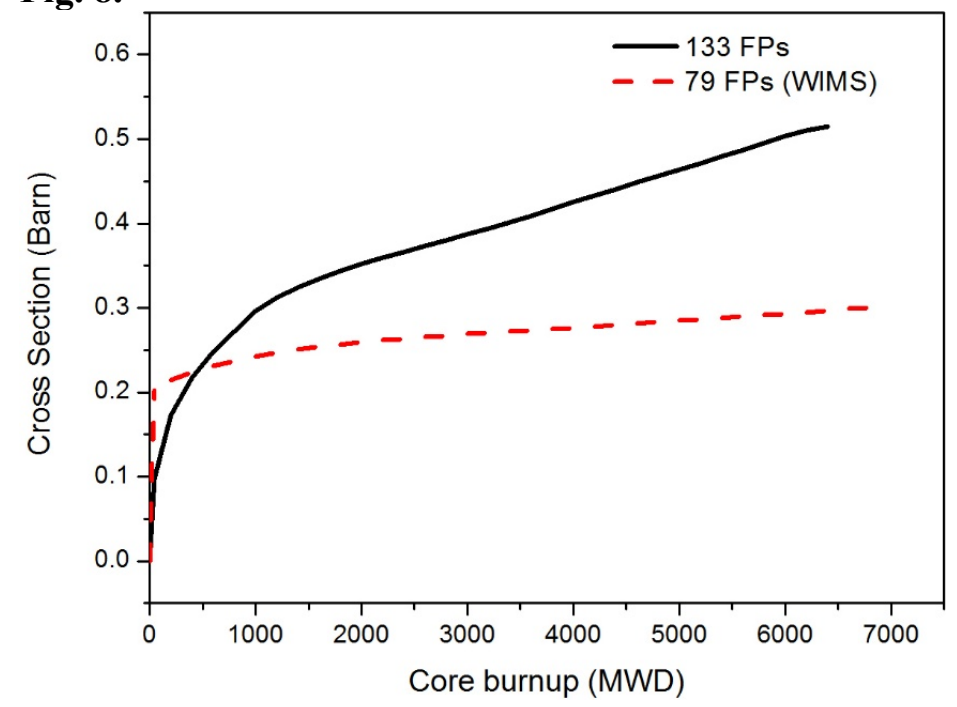


Fig. 9.

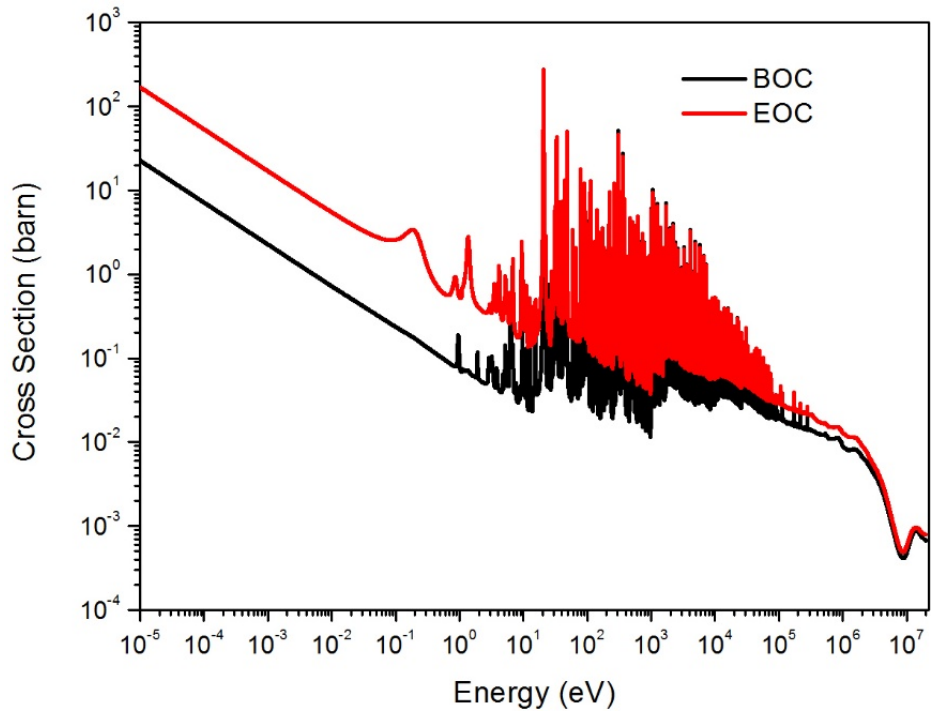

Fig. 10.

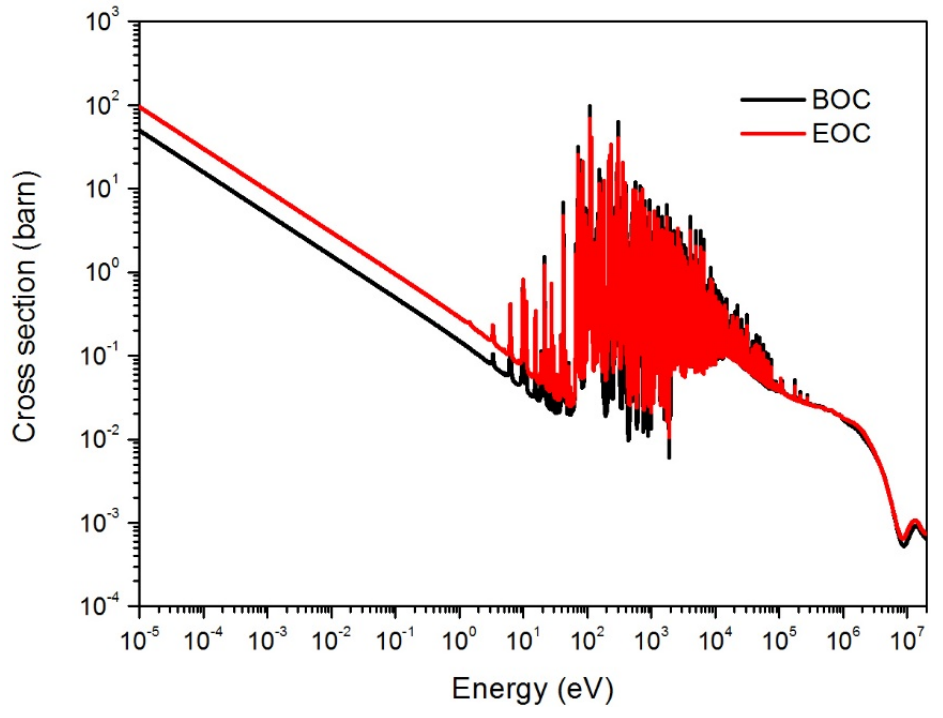


Table 1

\begin{tabular}{|c|c|c|c|c|c|c|c|c|c|}
\hline No.Nuclide & No.Nuclide & No.Nuclide & No. & Nuclide & No. Nuclide & No. Nuclide & No. Nuclide & No. & Nuclide \\
\hline $1 \mathbf{K r}-\mathbf{8 3}$ & $28 \mathrm{Ag}-109$ & 55 Ce-142 & 82 & Ga- 72 & $109 \mathrm{Sr}-88$ & $136 \mathrm{Cd}-112$ & $163 \mathrm{Sb}-127$ & 190 & Pm-150 \\
\hline $2 \mathbf{K r}-\mathbf{8 3 M}$ & 29 Cd-113 & $56 \mathrm{Ce}-143$ & 83 & Ge- 72 & $110 \mathrm{Sr}-89$ & $137 \mathrm{Cd}-114$ & $164 \mathrm{Sb}-128$ & 191 & Sm-150 \\
\hline $3 \mathbf{Y}-89$ & $30 \mathrm{Cd}-113 \mathrm{M}$ & 57 Ce-144 & 84 & Ge- 73 & $111 \mathrm{Sr}-90$ & $138 \mathrm{Cd}-115$ & 165 Te-122 & 192 & Sm-154 \\
\hline 4 Y-90 & 31 In-115 & 58 Pr-141 & 85 & Ge- 74 & 112 Zr-90 & $139 \mathrm{Cd}-115 \mathrm{M}$ & $166 \mathrm{Te}-124$ & 193 & Sm-156 \\
\hline $5 \quad \mathrm{Y}-91$ & 32 Te-127 & 59 Pr-143 & 86 & Ge- 76 & 113 Zr-92 & $140 \mathrm{Cd}-116$ & $167 \mathrm{Te}-125$ & 194 & Eu-152M \\
\hline 6 Zr-91 & 33 Тe-127M & 60 Pr-145 & 87 & As- 75 & 114 Zr-94 & $141 \mathrm{Cd}-117 \mathrm{M}$ & $168 \mathrm{Te}-125 \mathrm{M}$ & 195 & Eu-154 \\
\hline $7 \mathbf{Z r - 9 3}$ & 34 Te-129M & $61 \mathbf{~ N d - 1 4 3}$ & 88 & As- 76 & $115 \mathrm{Zr}-96$ & 142 In-113 & 169 Te-126 & 196 & Eu-157 \\
\hline $8 \mathbf{Z r - 9 5}$ & 35 I-129 & 62 Nd-144 & 89 & As- 77 & $116 \mathrm{Nb}-95 \mathrm{M}$ & 143 In-115M & 170 Te-128 & 197 & Eu-158 \\
\hline 9 Nb-93 & $36 \mathbf{I}-131$ & $63 \mathbf{~ N d - 1 4 5}$ & 90 & Se- 76 & $117 \mathrm{Nb}-96$ & 144 Sn-117 & 171 Te-130 & 198 & Gd-154 \\
\hline $10 \mathbf{N b}-\mathbf{9 3 M}$ & $37 \mathbf{I}-\mathbf{1 3 3}$ & 64 Nd-146 & 91 & Se-77 & 118 Мo-96 & $145 \mathrm{Sn}-117 \mathrm{M}$ & $172 \mathrm{Te}-131 \mathrm{M}$ & 199 & Gd-156 \\
\hline 11 Nb-95 & $38 \mathbf{I}-\mathbf{1 3 5}$ & 65 Nd-147 & 92 & Se-78 & 119 Mo-100 & 146 Sn-118 & $173 \mathrm{I}-127$ & 200 & Gd-158 \\
\hline 12 Mo-95 & 39 Xe-129 & 66 Nd-148 & 93 & Se-79 & $120 \mathrm{Ru}-100$ & 147 Sn-119 & $174 \mathrm{I}-130$ & 201 & Gd-159 \\
\hline 13 Мo-97 & 40 Xe-131 & 67 Nd-149 & 94 & Se- 80 & $121 \mathrm{Ru}-104$ & $148 \mathrm{Sn}-119 \mathrm{M}$ & $175 \mathrm{Xe}-128$ & 202 & Gd-160 \\
\hline 14 Мo-98 & 41 Xe-131M & 68 Pm-147 & 95 & Se- 82 & $122 \mathrm{Ru}-106$ & 149 Sn-120 & $176 \mathrm{Xe}-130$ & 203 & Gd-161 \\
\hline 15 Мo-99 & 42 Xe-132 & 69 Pm-149 & 96 & Br-79 & $123 \mathrm{In}-117 \mathrm{M}$ & $150 \mathrm{Sn}-121$ & 177 Xe-134 & 204 & Tb-159 \\
\hline 16 T-99 & 43 Xe-133 & 70 Pm-151 & 97 & Br-80M & 124 Sn-115 & $151 \mathrm{Sn}-121 \mathrm{M}$ & 178 Xe-136 & 205 & Tb-160 \\
\hline 17 T-99M & 44 Xe-133M & $71 \mathrm{Sm}-147$ & 98 & Br-81 & $125 \mathrm{Pd}-106$ & $152 \mathrm{Sn}-122$ & 179 Cs-134 & 206 & Tb-161 \\
\hline 18 Ru-99 & 45 Xe-135 & 72 Sm-149 & 99 & Br-82 & $126 \mathrm{Pd}-107$ & $153 \mathrm{Sn}-123$ & $180 \mathrm{Cs}-134 \mathrm{M}$ & 207 & Dy-160 \\
\hline 19 Ru-101 & 46 Xe-135M & 73 Sm-151 & 100 & $\mathrm{Kr}-82$ & 127 Pd-108 & 154 Sn-124 & $181 \mathrm{Cs}-136$ & 208 & Dy-161 \\
\hline $20 \mathrm{Ru}-102$ & 47 Cs-133 & 74 Sm-152 & 101 & $\mathrm{Kr}-84$ & 128 Pd-109 & 155 Sn-125 & 182 Cs- 137 & 209 & Dy-162 \\
\hline $21 \mathbf{R u}-103$ & 48 Cs-135 & 75 Sm-153 & 102 & $\mathrm{Kr}-85$ & $129 \mathrm{Pd}-110$ & $156 \mathrm{Sn}-126$ & 183 Ba-134 & 210 & Dy-163 \\
\hline $22 \mathbf{R h}-\mathbf{1 0 3 M}$ & 49 Ba-135 & $76 \mathbf{~ E u - 1 5 1}$ & 103 & $\mathrm{Kr}-86$ & $130 \mathrm{Pd}-111 \mathrm{M}$ & $157 \mathrm{Sb}-121$ & $184 \mathrm{Ba}-136$ & 211 & Dy-164 \\
\hline $23 \mathbf{R h}-\mathbf{1 0 3}$ & 50 Ba-137 & 77 Eu-153 & 104 & $\mathrm{Rb}-85$ & $131 \mathrm{Pd}-112$ & $158 \mathrm{Sb}-122$ & 185 Ba-138 & 212 & Dy-165 \\
\hline 24 Rh-105M & 51 Ba-140 & 78 Eu-155 & 105 & $\mathrm{Rb}-86$ & $132 \mathrm{Ag}-111$ & $159 \mathrm{Sb}-123$ & $186 \mathrm{Ce}-140$ & 213 & Ho-165 \\
\hline 25 Rh-105 & 52 La-139 & 79 Eu-156 & 106 & $\mathrm{Rb}-87$ & $133 \mathrm{Ag}-112$ & $160 \mathrm{Sb}-124$ & $187 \mathrm{Nd}-150$ & 214 & Ho-166 \\
\hline 26 Pd-105 & 53 La-140 & 80 Gd-155 & 107 & Sr-86 & $134 \mathrm{Ag}-113$ & $161 \mathrm{Sb}-125$ & 188 Pm-148 & & \\
\hline 27 Ag-107 & 54 Ce-141 & 81 Gd-157 & 108 & Sr-87 & $135 \mathrm{Cd}-111$ & $162 \mathrm{Sb}-126$ & 189 Pm-148M & & \\
\hline
\end{tabular}


Table 2

\begin{tabular}{ll}
\hline Reactor design description & \\
\hline Reactor type & Pool \\
Power level, MW & 10 \\
Number of standard fuel elements & 30 \\
Number of control fuel elements & 0 \\
Irradiation channels & 1 at core center \\
& 1 at core edge \\
Lattice pitch, mm ${ }^{2}$ & $77 \times 81$ \\
Fuel rod loading, g & 274 \\
Moderator, coolant, reflectors & Water \\
Burnup status of core & Initial core \\
Fuel element description & \\
\hline Fuel geometry & Rods \\
Fuel rods/standard Element & 16 \\
Fuel material & UZrH \\
Uranium enrichment, \% & 20 \\
Uranium density, g/cm & \\
Fuel rod outer diameter, mm & $3.72(45 \mathrm{wt} \% \mathrm{U})$ \\
Fuel rod active length, mm & 12.95 \\
Clad material & 558.8 \\
Clad outer diameter, mm & Incoloy 800 \\
Clad thickness, mm & 13.77 \\
Shroud material & 0.41 \\
Shroud side dimensions, mm & 2 \\
Shroud thickness, mm & Aluminum \\
Rod center to center spacing, mm & $75.72 \times 79.63$ \\
Rod-rod clearance, mm & 3.84 and 5.79 \\
Rod-shroud clearance, mm & 2.57 \\
Clearance between shrouds, mm & 1.37 \\
\hline & \\
\hline
\end{tabular}

Table 3

\begin{tabular}{l|ll}
\hline & BOC & Xe Equilibrium \\
\hline Current calculation (MCNP5) & $1.18624 \pm 0.0007$ & $1.14974 \pm 0.00051$ \\
MCNP6 & $1.18838 \pm 0.0005$ & $1.15319 \pm 0.00050$ \\
ANL (REBUS-2) & 1.1864 & 1.1544 \\
\hline
\end{tabular}

